\title{
Jalons vers Nulle Part : courte biochronologie de William Morris
}

Signposts to Nowhere: A short Bio-chronology of William Morris

\section{Gilbert Bonifas}

\section{(2) OpenEdition}

1 Journals

\section{Édition électronique}

URL : http://journals.openedition.org/rfcb/3238

DOI : $10.4000 /$ rfcb.3238

ISSN : 2429-4373

\section{Éditeur}

CRECIB - Centre de recherche et d'études en civilisation britannique

\section{Édition imprimée}

Date de publication : 1 septembre 2004

ISBN : 2-911580-19-2

ISSN : 0248-9015

\section{Référence électronique}

Gilbert Bonifas, " Jalons vers Nulle Part : courte biochronologie de William Morris », Revue Française de Civilisation Britannique [En ligne], XIII-1 | 2004, mis en ligne le 01 septembre 2004, consulté le 07 octobre 2019. URL : http://journals.openedition.org/rfcb/3238 ; DOI : 10.4000/rfcb.3238

Ce document a été généré automatiquement le 7 octobre 2019.

\section{cc) (†)}

Revue française de civilisation britannique est mis à disposition selon les termes de la licence Creative Commons Attribution - Pas d'Utilisation Commerciale - Pas de Modification 4.0 International. 


\section{Jalons vers Nulle Part : courte biochronologie de William Morris}

Signposts to Nowhere: A short Bio-chronology of William Morris

\section{Gilbert Bonifas}

Caricature parue dans Funny Folks à la suite des incidents de Dod Street le 20 septembre 1886.

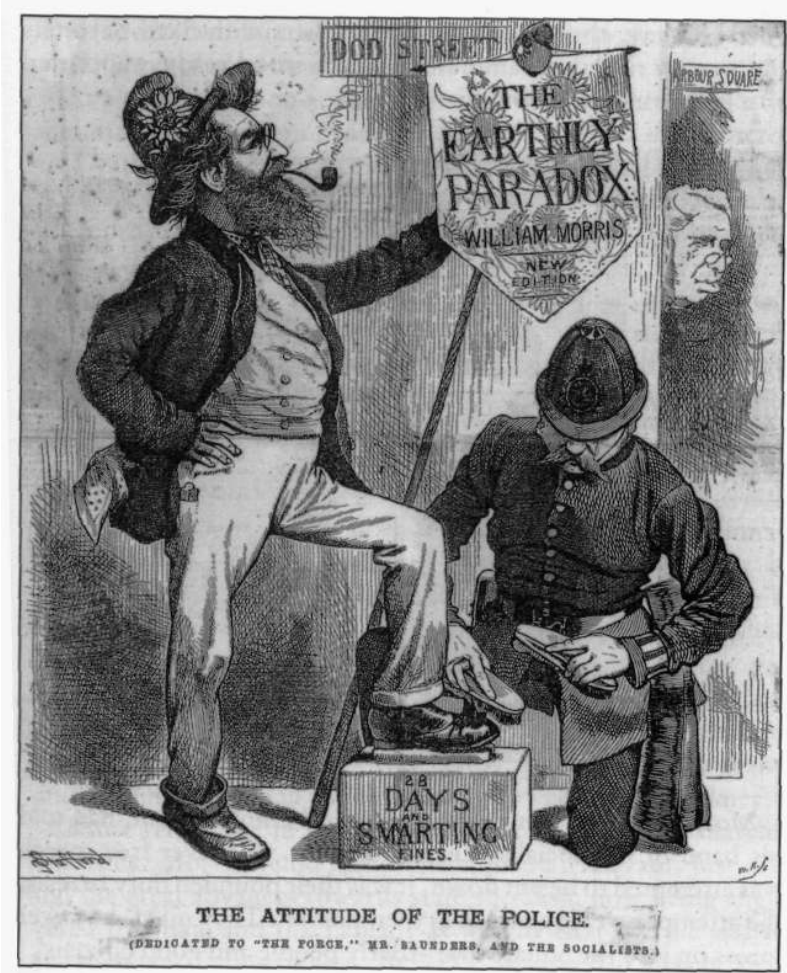

Le juge Saunders (à droite) vient de relaxer William Morris, accusé de rébellion contre agent de la force publique, alors qu'il a condamné les manifestants inconnus à de lourdes peines (sur la caisse à cirage). À la question du juge, 'What are you?', Morris avait répondu sans aucune fausse modestie : 'l am an artist, and a literary man, pretty well known, I think, throughout Europe'. 
(

1 1833 : William Morris père (né en juin 1797), associé principal dans une firme de courtage de la Cité de Londres, Sanderson \& Co, quitte son appartement de Lombard Street pour s'installer avec son épouse et ses deux premiers enfants à Walthamstow, alors village paisible en bordure de la forêt d'Epping. C'est là que naît le $\mathbf{2 4}$ mars 1834 William Morris. Six autres frères et sœurs suivront. En 1840, la famille Morris, très à l'aise, s'éloigne un peu plus de Londres et loue ( $£ 600$ par an) Woodford Hall dans l'Essex, grande demeure de style palladien entourée de tous côtés par la forêt d'Epping. La propriété possède son propre parc de 25 hectares et une ferme d'une cinquantaine d'hectares. C'est dans ce cadre champêtre, et à certains égards quasi féodal ${ }^{1}$, que le jeune Morris va apprendre à aimer la nature et s'éprendre du passé. Il chevauche son poney revêtu d'une armure, explore la forêt d'Epping et les vieux manoirs de l'Essex, est fasciné par les plaques mortuaires des églises de l'Essex. En 1844 son père l'amène visiter la cathédrale de Cantorbéry ; ce sera l'un des épisodes marquants de son enfance qu'il évoquera encore avec émotion plus d'un demi-siècle plus tard. Entre temps, il lit les romans de Walter Scott et plus tard assurera les avoir tous finis en 1841. Ainsi que l'écrit J.W. Mackail, vers le milieu des années 1840, superficiellement du moins, 'the love of the Middle Ages was born in him'. ${ }^{2}$ William Morris père meurt brutalement le $\mathbf{8}$ septembre 1847. Sa succession est évaluée à $£ 60$ 000. La famille quitte Woodford Hall pour une maison plus petite, Water House, à Walthamstow, mais continue de vivre très confortablement.

\section{(II)}

2 1848. Morris est admis à la public school de Marlborough College où il demeurera jusqu'en novembre 1851, ne montrant pendant tout ce temps qu'un goût modéré pour les études. Il préfère écumer la bibliothèque, où il lit tous les ouvrages traitant d'architecture et d'archéologie, et visiter les vieilles églises du Wiltshire ainsi que les sites préhistoriques de la région. Selon Jeffrey Spear, 'Morris carried forward in his life and art patterns established in childhood. His love of the English landscape and romance narratives, fused in imagination with his ever deepening knowledge of the Middle Ages, carried him out of an uncongenial present as a child and as a man'. ${ }^{3}$ En janvier 1853 Morris entre à Exeter College, Oxford. La ville est encore, malgré l'arrivée du chemin de fer dans ses faubourgs, pour l'essentiel une cité médiévale. Il se lie d'amitié avec Edward BurneJones qui restera son ami le plus proche jusqu'à la fin de ses jours. Avec quelques autres undergraduates ils forment un petit groupe ('the Brotherhood') qui cesse bientôt de discuter de religion pour s'intéresser à la littérature et aux chroniques médiévales. À la Bibliothèque bodléienne Morris se plonge dans les études sur l'architecture gothique et se penche sur les manuscrits enluminés du Moyen Âge. En 1853 il lit Past and Present, que Carlyle avait publié en $\mathbf{1 8 4 3}$. Cette représentation contrastée de l'univers harmonieux, solidaire et humain d'un monastère du douzième siècle et de l'égoïsme, du tumulte et de la cruauté du monde bourgeois contemporain lui apparaît 'as inspired and absolute truth'4. La même année, ou peut-être en 1854, il prend connaissance "avec un enthousiasme passionné» (Paul Meier) des deux premiers tomes de Modern Painters de Ruskin initialement publiés en 1843 et 1846. Dans la foulée suit la lecture d'autres 
œuvres de Ruskin : The Seven Lamps of Architecture (première publication en 1848) et The Stones of Venice (trois tomes publiés entre mars 1851 et juin 1853), qui affirment que le tempérament moral d'un peuple se reflète dans son architecture et glorifient l'art gothique. Le chapitre sur 'The Nature of Gothic' dans The Stones of Venice, sous-tendu par l'idée que l'art n'est rien d'autre que la résultante du plaisir que l'ouvrier éprouve dans son travail, allait avoir une influence cruciale sur le développement ultérieur de l'œuvre et de la pensée de Morris en lui permettant d'ordonner ses impressions et ses idées sur le Moyen Âge. En 1854, enfin, Morris apprend, en lisant Ruskin ${ }^{5}$, l'existence des préraphaélites ${ }^{6}$ et de leur retour à la manière des maitres du Moyen Âge. Son attirance pour le gothique s'en trouve renforcée et, en quelque sorte, légitimée.

(III)

3 En août 1854 Morris visite la Belgique et le nord de la France. Il découvre la peinture des Van Eyck et de Memling et est saisi d'admiration devant les splendeurs gothiques des cathédrales d'Amiens, Beauvais, Chartres et Rouen. En mars 1855 il hérite d'une partie des actions de son père. Jusqu'en 1872 elles lui rapporteront au total $€ 8803$, somme considérable qui allait lui permettre de ne prendre sérieusement en main la direction de Morris, Marshall, Faulkner \& Co (voir infra [V]) qu'à partir des années 1870 et de se consacrer auparavant à l'architecture, la littérature et la peinture ${ }^{7}$. Lors de l'été 1855 Morris visite à nouveau, en compagnie de Burne-Jones, vingt-quatre églises et neuf cathédrales du nord de la France dont Amiens, Chartres et Rouen. Lors de leur passage à Paris, dans une exposition consacrée à la peinture anglaise, ils sont ravis de voir figurer sept toiles préraphaélites. Au retour, sur les quais du Havre, les deux jeunes gens décident de ne pas entrer dans les ordres et de consacrer leur vie à l'art ${ }^{8}$; Morris envisage de devenir architecte. À l'automne de 1855 il obtient son diplôme d'Oxford.

\section{(IV)}

En 1856, par l'intermédiaire de Burne-Jones à qui il rend fréquemment visite à Londres, Morris fait la connaissance de Rossetti. À la fin de l'été, il s'installe dans la capitale et peu après, sous l'influence de Rossetti, renonce à son apprentissage d'architecte chez G.E. Street, l'un des principaux artisans du Gothic Revival, pour s'essayer à la peinture et à l'écriture (son premier recueil de poèmes, The Defence of Guenevere and other poems, tous d'inspiration médiévale, paraîtra en mars 1858). Il continue de lire abondamment et découvre les Rural Rides de Cobbett (1830), autre démonstration de la supériorité du Moyen Âge sur les temps modernes. Il finira par connaître l'ouvrage quasiment par cœur et utilisera lui-même fréquemment la métaphore de 'the great wen' pour faire référence à Londres. À l'automne 1857 Morris rencontre Jane Burden, fille d'un palefrenier et modèle de Rossetti, dont les traits étaient le beau idéal du préraphaélitisme. Le 24 avril 1859 Morris l'épouse. Le mariage se révélera vite être un échec. 
( $)$

5 Avec la collaboration de son ami Philip Webb, architecte, Morris entreprend en juin 1859 la construction (pour $£ 4000$ ) de Red House, vaste demeure aujourd'hui encerclée par Bexleyheath, dont la décoration intérieure, assurée par Morris et ses fréquents visiteurs, incorpore de nombreux éléments de style gothique, des tentures aux fenêtres et à l'ameublement ${ }^{9}$. De là naquit l'idée, d'abord suggérée par Ford Madox Brown, de fonder une entreprise de décoration dans laquelle, comme au Moyen Âge, les artistes eux-mêmes participeraient à la production au lieu de l'abandonner à des ouvriers fabriquant en masse des objets sans valeur esthétique ${ }^{10}$. Le 11 avril 1861 est donc créé Morris, Marshall, Faulkner \& Company, 'Fine Art Workmen in Painting, Carving, Furniture and the Metals' selon le prospectus diffusé alors. Outre Morris les membres fondateurs étaient Ford Madox Brown, Burne-Jones, Rossetti, Philip Webb, P.P. Marshall et Charles Faulkner. Morris fut nommé directeur; d'après Rossetti 'not because we ever dreamed he would turn out to be a man of business, but because he was the only one of us who had time and money to spare'. La firme installe ses ateliers en plein cœur de Londres, à Red Lion Square. Grâce à l'engouement de l'époque pour le Moyen Âge, au Gothic Revival et, les premières années, à la construction incessante de nouvelles églises anglicanes nécessitant de nombreux vitraux (dont la fabrication devint l'activité essentielle de la firme), les commandes ne manquèrent pas. Cependant, en raison de la concurrence et d'une gestion parfois hasardeuse, les bénéfices ne furent pas énormes et la plupart des associés de Morris se tournèrent également vers d'autres activités. Lui, au contraire, s'absorba de plus en plus dans la gestion et la production de Morris, Marshall, Faulkner \& Co, de sorte que, lorsqu'en 1869 et 1870 la valeur de ses actions s'effondra, il prit conscience que la firme devait désormais, pour survivre (et lui assurer des revenus corrects), être gérée de façon plus commerciale et s'étendre. Au début des années 1870, par de nouvelles créations (papiers peints, tissus d'ameublement) Morris atteignit une clientèle fortunée et en août 1874 annonça à ses associés qu'il souhaitait reprendre l'affaire à son compte. Le $\mathbf{3 1}$ mars $\mathbf{1 8 7 5}$ Morris devient l'unique propriétaire de la société dont la raison sociale se change en Morris \& Company. L'entreprise ne va plus cesser de s'étendre et de prospérer, laissant Morris libre, à partir de 1891, de se consacrer à la typographie en fondant la Kelmscott Press qui devint vite, également, une entreprise florissante ${ }^{11}$. De la sorte, à partir des années 1880, lorsque son engagement politique se radicalisa, Morris se crut à diverses reprises obligé d'expliquer comment l'on pouvait à la fois être homme d'affaires prospère et socialiste révolutionnaire $^{12}$. Son principal argument ${ }^{13}$ fut toujours que quoi qu'il fit personnellement pour aller à l'encontre de la logique du système, cela ne contribuerait nullement à renverser le capitalisme, seul objectif qui importait.

(VI)

6 En mai 1871 Morris découvre Kelmscott Manor et juge la demeure 'a heaven on earth ; an old stone Elizabethan house... and such a garden! close down on the river, a boat house and all things handy. ${ }^{14}$ Le mois suivant Morris et Rossetti louent Kelmscott Manor et le domaine environnant. Le 9 juillet, Morris dont l'intérêt pour les sagas scandinaves remonte à sa lecture de la Northern Mythology de Benjamin Thorpe (1851-1852) ${ }^{15}$ à Oxford en 1853 et a été réveillé par sa rencontre avec un lettré islandais de Cambridge, 
Eirikr Magnüsson, appareille d'Édimbourg pour l'Islande, laissant Jane et ses deux filles à Kelmscott Manor en compagnie de Rossetti ${ }^{16}$. Morris fera un second séjour en Islande en juillet 1873. De l'avis de plusieurs de ses biographes, ces deux voyages auront sur lui un effet roboratif, physiquement et plus encore spirituellement. La vue des Islandais faisant front, grâce à leur solidarité et un fort sentiment d'appartenance communautaire, dans un milieu rude et pauvre, l'impression qu'ils formaient un peuple libre, ignorant des inégalités sociales, semblent avoir instillé en Morris des idéaux oubliés ${ }^{17}$ ou des convictions nouvelles ${ }^{18}$ et lui avoir permis d'échapper à l'emprise esthétique de Rossetti et à la tentation de l'art pour l'art. A partir du milieu des années 1870 il s'intéresse de manière de plus en plus engagée aux affaires du monde, se lançant en toutes circonstances dans des tirades contre les horreurs de la civilisation moderne et commençant à rêver fréquemment d'une autre société. Dès le 26 mars 1874 il écrit dans une lettre :

Now it seems to be nobody's business to try to better things... But look, suppose people lived in little communities among gardens and green fields, so that you could be in the country in five minutes' walk, and had few wants... and studied the (difficult) arts of enjoying life, and finding out what you really wanted: then I think one might hope civilisation had really begun. ${ }^{19}$

\section{(VII)}

7 De $\mathbf{1 8 7 6}$ à 1878, des "horreurs bulgares " au Congrès de Berlin, Morris participe activement à la campagne de protestation contre les massacres turcs en Bulgarie et ensuite contre la politique du gouvernement de Disraeli qui menaçait la Russie d'une guerre si elle attaquait l'empire ottoman. Ce faisant, Morris se rapproche de certains cercles radicaux londoniens et s'éloigne des libéraux et de Gladstone, finissant par considérer que leurs prises de position contre l'oppression dont étaient victimes certains peuples en Europe étaient d'abord une stratégie pour susciter l'indignation morale du prolétariat anglais et le détourner ainsi du combat contre les injustices économiques et pour une société meilleure en Grande-Bretagne. Morris, en effet, est désormais convaincu, après avoir été le témoin de la mobilisation des ouvriers anglais contre la politique pro-turque de Disraeli, que la classe ouvrière britannique est potentiellement révolutionnaire et son Manifesto to the Working Men of England de mai 1877 fut probablement son premier effort pour éveiller la conscience politique du prolétariat et l'avertir que si on la laissait parvenir au pouvoir, 'a certain part of the richer classes of England would thwart your just aspirations, would silence you, would deliver you bound hand and foot forever to irresponsible capital'.

\section{(VIII)}

En 1877, Morris avait également fondé The Society for the Protection of Ancient Buildings (très vite connue sous le nom de The Anti-Scrape) non seulement pour empêcher la démolition d'édifices anciens, mais encore de douteuses "restaurations». Ce fut en grande partie afin de collecter des fonds pour l'Anti-Scrape que de 1877 à 1883 Morris multiplia discours et conférences ( 34 au total), traitant de la dégénérescence (voire de l'impossibilité) de l'art dans une société fondée sur le profit ; de la production de masse et de la division du travail ; de la fracture entre le beau et l'utile, le travail et le plaisir, depuis que l'artiste-artisan du Moyen Âge était devenu un prolétaire exploité à qui 
toute créativité était interdite; des classes moyennes en tant que suppôt de la civilisation du commerce, de la laideur, de la pollution et de la destruction de l'environnement par le capitalisme industriel. Morris rêve d'une cité-jardin, de la régénération de la société par l'établissement de l'égalité et de la solidarité, par l'extension de l'art à toutes les activités humaines, car sans «art populaire » il ne saurait y avoir de société véritablement civilisée. Ces textes ${ }^{20}$ sont fondamentaux pour comprendre la période militante de Morris. Ils contiennent déjà l'essentiel de son message, ce qui laisse songeur quant à l'influence exacte de la pensée marxiste sur Morris $^{21}$. Ils sont tous d'inspiration principalement ruskinienne ${ }^{22}$, à cette différence majeure près que Morris ne peut se contenter d'être critique; il lui faut agir, être "pratique $»^{23}$. De plus il rejette le salut par une nouvelle aristocratie comme le voudrait Carlyle, ou par le paternalisme dont la pensée de Ruskin est imprégnée, et si le Moyen Âge reste une référence constante, tout retour à une civilisation modelée sur le passé lui paraît impossible ${ }^{24}$. Ce qu'il nomme 'revolutionary politics' commence à lui sembler la seule option pertinente; en somme, la nostalgie doit devenir révolutionnaire et lorsqu'arrive l'été $\mathbf{1 8 8 2}$ il est prêt à s'allier à 'any body who distinctly called themselves Socialists' comme il l'écrit un an plus tard à Andreas Scheu ${ }^{25}$.

(IX)

9 Entre-temps Morris, dans le courant de l'été 1878, avait loué à Hammersmith 'The Retreat', plus tard rebaptisée Kelmscott House. La famille s'y installe en octobre. Du 10 au 16 août 1880, Morris, Jane et quelques amis remonteront la Tamise par petites étapes de Kelmscott House à Kelmscott Manor. Le 14 août Morris écrit : 'We were on the Thames that is the Thames, amidst the down-like country and all Cockneydom left far behind, and it was jolly'. Un voyage similaire aura lieu l'année suivante à la même époque.

\section{(X)}

En janvier 1883 Morris devient membre de la Democratic Federation présidée par Henry Mayers Hyndman. Morris et lui s'étaient rencontrés pour la première fois en 1879. En juin 1881 Hyndman avait publié England for All, qui l'avait brouillé avec Karl Marx (dont il avait fait la connaissance en 1880), deux chapitres étant des plagiats du Capital. À partir de cette date Morris devient un militant davantage préoccupé de promouvoir la révolution socialiste par des conférences incessantes aux quatre coins des îles Britanniques et la rédaction de très nombreux articles politiques que de poursuivre une œuvre artistique et littéraire, même s'il n'oublie pas totalement la poésie ${ }^{26}$ (pendant ses voyages il tue le temps en traduisant l'odyssée) et si parfois il dessine encore pour Morris \& Co de nouveaux modèles de papiers peints ou de textiles (la firme est alors efficacement gérée par George Wardle, son directeur administratif). Il commence aussi à lire Le Capital en français ${ }^{27}$, avec tant d'acharnement, semble-t-il, qu'il doit à nouveau donner son exemplaire à relier en 1884. Qu'il ait éprouvé beaucoup de peine à assimiler les parties non-historiques et techniques du livre ne fait aucun doute, mais il paraît probable (malgré les déclarations contradictoires de ses premiers biographes et de Morris lui-même, notamment sur son bon entendement de la théorie de la valeur et de la plus-value et sur l'importance de cette dernière pour le mouvement socialiste) qu'il fit de son mieux pour les comprendre. Il jugeait de telles lectures indispensables pour 
intellectualiser son sentiment d'injustice et son désir d'égalité et de fraternité ainsi que pour alimenter sa réflexion sur la société capitaliste. On suivra Paul Meier lorsqu'il écrit que "dès ce moment, les allusions au Capital et, plus encore, les emprunts se multiplient dans son ceuvre ${ }^{28}$ et il est fort possible que sans ses lectures de Marx, Morris n'aurait pu écrire (avec Hyndman) A Summary of the Principles of Socialism en $\mathbf{1 8 8 4}$ et (avec Belfort Bax) 'Socialism fom the Roots Up', série d'articles rédigés pour Commonweal en 1886-1888, et Socialism, its Growth and Outcome en 1893. Il est moins certain que Le Capital était un préalable indispensable à la rédaction de News from Nowhere ${ }^{29}$ et il n'y a pas lieu de minimiser, comme source d'inspiration, l'Utopie " communiste » de Thomas More que Morris avait lue bien avant Le Capital (et relue en 1882) et qu'il considérait comme un prototype de la société nouvelle dont il souhaitait l'avènement. Dans l'introduction qu'il rédigea en $\mathbf{1 8 9 3}$ pour l'édition Kelmscott de l'Utopie, Morris ne place-t-il pas ce dernier (et lui-même), par ses références historiques, dans la lignée de tous ceux qui ont rêvé une société idéale où règne l'égalité ${ }^{30}$ ?

Figure 1 : Carte d'adhérent de la Democratic Federation, dessinée par William Morris en 1883.

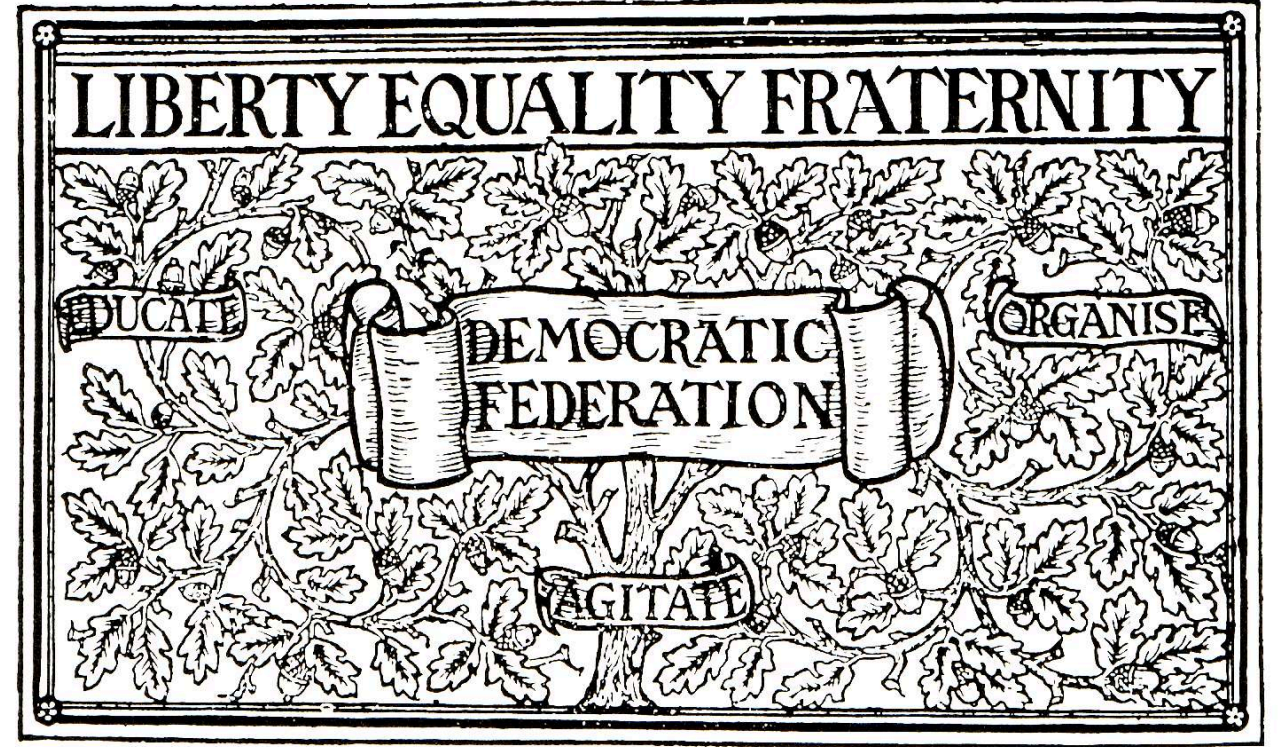

(XI)

11 Le 14 juin 1884 la section de Hammersmith de la Democratic Federation se constitue à Kelmscott House, qui va devenir son lieu de réunion habituel. À la fin de $\mathbf{1 8 8 4}$ elle comptera 39 membres. Le 4 août $\mathbf{1 8 8 4}$ la Democratic Federation devient la Social Democratic Federation (SDF), mais depuis quelques mois Morris est inquiet. L'opportunisme et l'autocratisme de Hyndman l'indisposent, tout comme son désir de faire de la SDF un parti orthodoxe qui ferait campagne pour des réformes spécifiques et présenterait des candidats aux élections, tout en continuant, paradoxalement, à prêcher une impossible révolution immédiate et à prôner un socialisme qui, aux yeux de Morris, ne pouvait être qu'un socialisme d'État de type bismarckien, et donc un nouveau mode d'oppression. Lui-même considère alors que la révolution ne pourra se produire qu'après que la classe ouvrière aura été éduquée dans les principes du socialisme et amenée à vouloir un changement radical de société31. Finalement, le 27 
décembre 1884, Morris et ses amis (dont Eleanor Marx, Edward Aveling et Belfort Bax) quittent la SDF et le $\mathbf{3 0}$ décembre fondent la Socialist League. Le manifeste publié en janvier 1885 est étroitement basé sur Le Capital. Le $\mathbf{2 8}$ janvier paraît, en grande partie financé par Morris, le premier numéro de Commonweal. Kelmscott House est désormais le siège de la section de Hammersmith de la Socialist League, vite dénommée par les autres membres de la League 'the damned bourgeois branch'.

\section{(XII)}

De février 1885 à la fin de 1890, Morris va plus que jamais mettre son énergie naturelle et sa très grande capacité de travail au service du socialisme, conformément au mot d'ordre inscrit sur la carte de membre de la Democratic Federation que lui-même avait dessinée deux ans auparavant, en 1883 : 'Agitate, Educate, Organise' (voir fig. 1). L'époque se prêtait au militantisme. La seconde moitié des années 1880 fut caractérisée par une grave récession économique entrânant, surtout parmi les ouvriers les moins qualifiés, chômage et disette et un mécontentement qui se traduisit à plusieurs reprises par des manifestations de masse et des heurts sérieux avec la police, notamment le $\mathbf{8}$ février 1886 ('Black Monday') et le 13 novembre 1887 ('Bloody Sunday') (voir fig. 2).

Figure 2 : 'Bloody Sunday' : la bataille de Trafalgar Square, 13 novembre 1887.

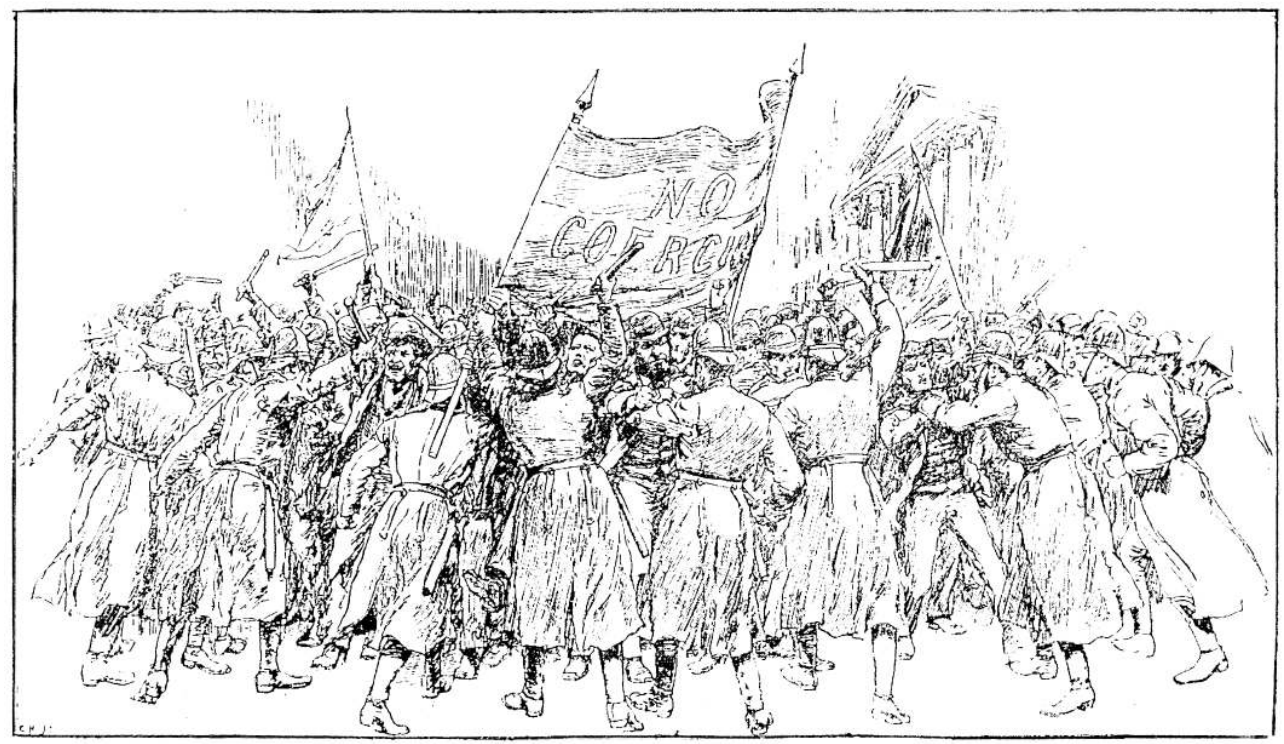

13 On verra donc Morris haranguer les foules aux coins des rues, prendre la tête de défilés ouvriers, se faire arrêter deux fois par la police (et chaque fois, étant un gentleman, être traité avec grande indulgence par le juge), parcourir l'Angleterre pour donner des conférences et encourager la création de sections locales de la Socialist League. Il sera aussi le directeur de Commonweal et y contribuera sans discontinuer éditoriaux et articles où il peaufinera la définition de la révolution sociale et de la société à venir qu'il avait donnée dans son premier article pour le journal en 1887, 'How We Live and How We Might Live'32. 
(XIII) profondément divisés entre les partisans d'une participation au système politique en place, les tenants d'un socialisme étatique et des anarchistes qui posaient volontiers aux dinamiteros et qui, à partir de $\mathbf{1 8 8 9}$, devinrent prépondérants, de nombreux " parlementaristes » ayant abandonné la Ligue entre 1887 et 1889. Au congrès annuel du parti, en mai 1890, Morris perdit définitivement le contrôle de la Socialist League et fut contraint d'abandonner la direction de Commonweal. Le 21 novembre 1890 il quittait la Ligue et, suivi par les 120 membres de la section de Hammersmith (voir fig. 3), constitua avec eux (officiellement le 23 novembre 1890) The Hammersmith Socialist Society. ${ }^{37}$ Son manifeste rejetait à la fois le parlementarisme et l'anarchisme et définissait son rôle comme étant de répandre les principes du socialisme 'by Lectures, Street meetings, and Publications ... and that object only'. Malgré cela Morris ne renonça pas à militer pour le socialisme et autant que sa santé désormais chancelante le lui permettait, continua de manifester, d'écrire et de parler en sa faveur. Mais ses efforts pour unir son propre « parti », la SDF et les Fabiens se soldèrent par un échec en $\mathbf{1 8 9 3 .}$ 
Figure 3 : Carte d'adhérent de la section de Hammersmith de la Socialist League, dessinée par Walter Crane. On notera l'étrange ressemblance entre le forgeron de l'image et l'apparence physique du trésorier. Sur le secrétaire, voir « William Morris et les arts du livre » infra.

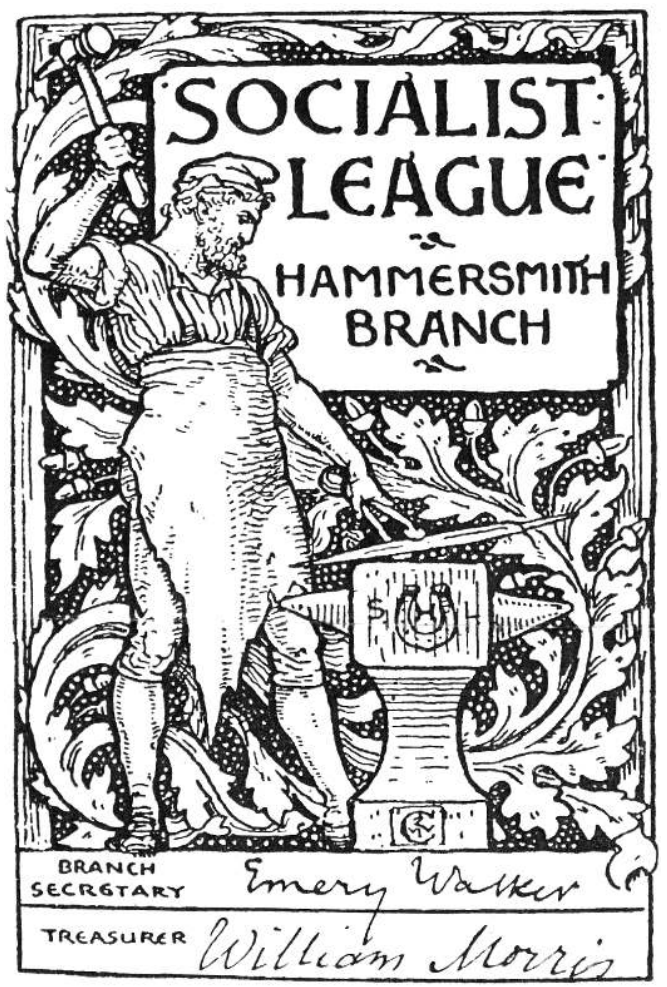

18 Les dernières années de sa vie il se rapprocha à nouveau de la $\mathrm{SDF}^{38}$, concédant même que si les réformes demandées par cette dernière pour améliorer le sort des ouvriers n'étaient que des «mesures palliatives ", elles pouvaient être néanmoins utiles dans le contexte de l'époque. Mais il resta jusqu'au bout convaincu qu'un socialisme authentique ne pouvait advenir que par l'entremise d'une révolution et un soir d' octobre 1895, après que Sidney Webb eut donné une conférence devant les membres de la Hammersmith Socialist Society, Morris lui déclara: 'The world is going your way at present Webb, but it is not the right way in the end'39. A cette date sa santé avait commencé à décliner rapidement. Une visite des fjords norvégiens (en juillet et août 1896), apparemment entreprise avec l'idée que l'air vif du grand Nord se révélerait roboratif ne l'améliora guère et il s'éteignit à Kelmscott House le 3 octobre 1896, d'une congestion pulmonaire et, pour ceux qui le connaissaient bien, aussi d'épuisement ${ }^{40}$.

(XV)

19 Combat culturel, l'éducation pour le socialisme et la révolution passait aussi par la littérature et pendant qu'il en fut le directeur (et propriétaire) Morris utilisa Commonweal à cet effet. En 1885-1886 parurent les poèmes qui finirent par constituer The Pilgrims of Hope dont Glasier dit qu'ils étaient 'a tale of frankly conventional love melodrama blended with the story of modern working-class revolt and the tragedy of the Commune of Paris... It is the first and as yet, so far as I know, the only poetical narrative of modern proletarian revolt which we possess ${ }^{41}$. De novembre 1886 à janvier 1887 parut également en feuilleton A Dream of John Ball (le livre sortit en 1888) qui relate la révolte paysanne de 1381 en Angleterre et illustre la théorie de Morris selon laquelle bien des 
gens sont par tempérament et inclination des socialistes qui s'ignorent, que cela est vrai depuis les débuts de l'histoire humaine et se vérifie particulièrement au Moyen Âge. A Dream of John Ball est un hymne à la fraternité et au sentiment d'appartenance communautaire, à la lutte pour la justice contre la tyrannie et une démonstration que les aspirations socialistes de la fin du dix-neuvième siècle plongent leurs racines dans le plus lointain passé national. Enfin c'est dans Commonweal que parut du $\mathbf{1 1}$ janvier 1890 au 4 octobre News from Nowhere. La première publication sous forme de livre eut lieu aux USA à la fin de 1890, selon le texte de Commonweal et sans l'autorisation de Morris. L'édition anglaise sortit en 1891 avec des modifications et des ajouts ${ }^{42}$.

\section{$(\mathrm{XVI})$}

20 Les sources de News from Nowhere, certaines, plausibles, spéculatives, ayant été minutieusement répertoriées et soupesées ${ }^{43}$, il n'y a pas lieu de s'attarder. L'on se contentera ici d'attirer l'attention sur quelques points. L'on rappellera d'abord que ce qui déclencha la rédaction du roman fut la publication et l'énorme succès, en Angleterre aussi bien qu'aux États-Unis, de l'utopie d'Edward Bellamy, Looking Backward (1888), qui dépeignait une société centralisée, étatisée, embrigadée, mécanisée et urbanisée à l'extrême, opposant travail et plaisir, montant en épingle tout ce que Morris détestait ${ }^{44}$. Mais plus encore que la révulsion, ce qui motiva Morris, ce fut la crainte de voir les socialistes anglais déserter ce que lui considérait comme le seul socialisme authentique ${ }^{45}$. Ainsi News from Nowhere serait la manifestation d'une double révolte : contre la société victorienne et l'aliénation qu'elle génère, et contre un certain socialisme ${ }^{46}$.

\section{$(\mathrm{XVII})$}

21 En second lieu on doit s'interroger sur la nature politique exacte de la société décrite dans Nouvelles de Nulle part. Il se peut qu'elle soit une "vision » de la phase ultime du communisme, mais, comme le souligne Lyman Tower Sargent ${ }^{47}$, peu d'anarchistes trouveraient à redire au tableau que donne Morris d'un monde où la liberté individuelle a été étendue autant qu'il est possible, où la contrainte et la coercition ont été réduites au maximum, où il ne semble plus y avoir d'État central et où, pour tout ce qui concerne la vie en commun, l'on essaie de parvenir à un consensus. Sur ces divers points les projets communiste et anarchiste se recoupent et même si Morris dépensa beaucoup d'énergie à combattre les anarchistes de la Socialist League ${ }^{48}$ et condamna l'anarchisme comme doctrine politique, l'on ne peut écarter la possibilité d'une influence de celui-ci sur News from Nowhere. Sargent ajoute qu'il y avait une fibre anarchiste chez Morris $^{49}$ et qu'elle se trouva renforcée par sa fréquentation de Kropotkine $^{50}$. On trouve en effet, dans la conception que Kropotkine se fait de la bonne société, une réconciliation des pôles ville/campagne, travail/plaisir, discipline/ autonomie, production/consommation qui a son symétrique dans le roman de Morris ${ }^{51}$. De sorte que John Payne se croit autorisé à conclure : 'For all that Morris read Marx... and called himself a communist, it is the communitarian strand of nineteenth century thinking (from Blake via Christian Socialism, Tolstoy and Kropotkin) that the lyricism of News from Nowhere most strongly brings to mind ${ }^{52}$. 


\section{(XVIII)} utopique de la fin du siècle. Le roman est à l'évidence le produit d'un esprit utopique tel que le définit Karl Mannheim: 'A state of mind is utopian when it is incongruous with the state of reality within which it occurs ${ }^{153}$ et très classiquement on peut rendre compte de sa genèse en faisant référence à la fois à la quête, semble-t-il éternelle chez l'homme, d'un paradis perdu et au milieu politique et économique dans lequel son auteur était contraint de vivre. Les sociétés idéales imaginaires ${ }^{54}$ sont souvent les conséquences littéraires de temps mauvais; en raison du contexte social, les vingt dernières années $\mathrm{du}$ dix-neuvième siècle furent particulièrement prolifiques en récits utopiques proposant des solutions alternatives à divers maux affectant la société britannique. Le contre-projet de Morris fut une des expressions de cette dissidence qui contestait la civilisation et l'ordre victoriens en reconstruisant l'espace ou en accélérant le temps. Il y voisine avec le After London, or Wild England de Richard Jefferies dont on sait que Morris le lut peu après sa parution en avril 1885 avec délectation car il s'agit du roman de la mort de l'Angleterre industrielle et capitaliste. Ravagé par un cataclysme, le pays est retourné à l'état sauvage. Toute trace de progrès technique a disparu ; Londres n'est plus que ruines et s'enfonce dans la Tamise ; marécages, forêts, broussailles recouvrent tout ; les survivants sont revenus à la barbarie, c'est-à-dire à une existence plus brutale, mais aussi plus simple et moins corrompue. On cite parfois After London parmi les sources de News from Nowhere. Il y a certes des correspondances ${ }^{55}$, mais si influence il y eut, elle fut surtout négative : ce qui importe, c'est que Morris parvint à surmonter sa fascination pour l'apocalypse ${ }^{56}$ et comprit que le salut ne pouvait résider dans une régression vers le primitivisme. Cela étant, After London et News from Nowhere, chacun à sa manière, s'inscrivent dans le courant du retour à la nature qui court en filigrane à travers toute l'histoire intellectuelle et sociale du dix-neuvième siècle et qui, articulé souvent sur une image idéalisée du passé, contribua à la lutte contre une société industrielle et marchande. À partir des années 1880 l'idée du retour à la nature se transforma plus généralement en idéal de la vie simple. Le mouvement ouvrier, préoccupé par l'amélioration des conditions de vie matérielles du prolétariat, n'y porta guère d'intérêt, mais il figure à l'évidence chez Morris et chez tous ceux, "les socialistes éthiques ", qui estimaient que ce à quoi il fallait d'abord échapper était la pauvreté spirituelle du capitalisme.

De la sorte News from Nowhere, par bien des aspects (l'appréhension sensuelle du monde, un certain anti-intellectualisme, le refus d'exploiter la nature, une atmosphère quasiment adamique), appartient aussi à ce que Peter C. Gould a qualifié de 'early green politics ${ }^{57}$ et Morris aura une influence capitale sur Robert Blatchford, qui fit de la vie simple et naturelle (ainsi que de l'ouverture de la classe ouvrière à l'art et à la beauté) un concept central de son socialisme tel qu'on le trouve exposé à la fois dans son journal, le Clarion, dans Merrie England (1893), recueil de ses articles du Clarion ${ }^{58}$, et dans The Sorcery Shop (1907), quasi-remake de News from Nowhere ${ }^{59}$.

\section{$(\mathrm{XIX})$}

Tentative pour substituer un autre imaginaire à celui du capitalisme et donc renverser les mentalités, News from Nowhere n'est pas à prendre au pied de la lettre, sans quoi avec

Revue Française de Civilisation Britannique, XIII-1 | 2004 
son économie politique digne de cocagne, il ne pourrait au mieux être qu'un jeu d'esprit ${ }^{60}$, au pire un livre "fade et charmeur » ne dépeignant qu'une "vaste bergerie " ${ }^{61}$. Inséré dans le combat culturel que mène Morris contre une vision bourgeoise du monde et l'empire de la technique et de l'économie, Nouvelles de Nulle Part devient un discours de rupture avec l'idéologie en place et tire sa force non pas d'une construction intellectuelle cohérente et rationnelle, mais de sa capacité lyrique et de sa dimension mythique $^{62}$. Eugene D. LeMire n'a vraisemblablement pas tort de conclure que News from Nowhere, par delà son évocation d'une époque de repos, quelque part vers la fin de l'Histoire, se voulait d'abord un appel à la résistance et à l'action ${ }^{63}$. De ce point de vue, on notera pour finir les tentatives faites récemment pour attribuer à News from Nowhere un rôle culturel actif dans le combat mené contre la modernité capitaliste en présentant le livre comme 'a vision for our time $e^{\text {'64 }}$ et en en faisant un œuvre émancipatrice ${ }^{65}$. Ainsi Nouvelles de Nulle Part poursuit sa carrière dans le mouvement des idées alors qu'on aurait pu le croire depuis longtemps cantonné dans leur histoire ${ }^{66}$.

\section{NOTES}

1. Voir la description de la vie quotidienne à Woodford dans J.W. MACKAIL, The Life of William Morris, London: Longmans, Green \& Co, 1901, vol. 1, p. 9.

2. Ibid., p.10

3. Jeffrey C. SPEAR, Dreams of an English Eden : Ruskin and his Tradition in Social Criticism, New York: Columbia University Press, 1984, p.206.

4. J.W. MACKAIL, The Life of William Morris, vol. 1, p. 38.

5. Qui défendait cette rupture avec l'académisme dans ses Lectures on architecture and painting delivered at Edinburgh in November 1853. L'ouvrage parut en 1854.

6. La Confrérie préraphaélite (the Pre-Raphaelite Brotherhood) avait été fondée en septembre 1848 par sept jeunes hommes, dont John Everett Millais, William Holman Hunt et Dante Gabriel Rossetti.

7. Pour plus de détails voir le chapitre 2 ('The Origins of the Morris Family Fortune') de Charles HARVEY \& John PRESS, Art, Enterprise and Ethics : The Life and Work of William Morris, London: Frank Cass, 1996, pp. 28-44.

8. Sur l'art de Morris et les liens avec sa pensée, lire dans le présent numéro, pp. 123-133, Michel REMY, « La perspective prélapsarienne de William Morris »

9. À Webb il avait demandé de lui bâtir 'a house very medieval in spirit' et une fois la maison construite estima avec satisfaction qu'elle était dans 'the style of the thirteenth century' (cité par Paul THOMPSON, The Work of William Morris, Oxford: Oxford University Press, 1991, pp. 13 et 14).

10. Idéal qui ne fut jamais que très partiellement réalisé, mais qui ne fut jamais totalement oublié non plus et permit à la fois d'éviter les dérives commerciales qu'encourageait un marché en expansion et de résister à la tentation de recourir plus que nécessaire à la machine.

11. Voir dans le présent numéro, pp. 135-156, l'article d'Antoine CAPET, « William Morris et les arts du livre ".

12. Lui-même offrit toujours à ses employés des conditions de travail et des salaires plus généreux que ce que les lois du marché semblaient alors justifier. Selon HARVEY \& PRESS, Art, 
Enterprise and Ethics, pp. 194-215, la gestion de Morris \& Co releva toujours d'une éthique ruskinienne.

13. Pour plus de détails voir notamment Paul MEIER, La pensée utopique de William Morris, Paris : Éditions sociales, 1972, pp. 74-80 ; Anna von HELMHOLTZ-PHELAN, The Social Philosophy of William Morris, Durham (N.C.): Duke University Press, 1927, pp. 76-81 et J.W. MACKAIL, The Life of William Morris, vol. 2, pp. 134-139.

14. Philip HENDERSON (ed.), The Letters of William Morris to his Family and Friends, London: Longmans, Green \& Co, 1950, p. 41.

15. Le titre complet étant Northern Mythology, comprising the principal popular traditions and superstitions of Scandinavia, North Germany, and the Netherlands. Compiled from original and other sources.

16. On ne s'attardera pas ici sur la «liaison» de Jane Morris avec Rossetti, dont on ne sait d'ailleurs si elle en fut réellement une (sur ce point voir les remarques judicieuses de Paul THOMPSON, The Work of William Morris, pp.23-30). La séparation affective de Morris et de sa femme se précisa à l'automne 1865 lorsqu'ils quittèrent Red House pour aller habiter à Londres au-dessus des ateliers de la firme (alors au 26, Queen Square). C'est un peu plus tard que Jane commença de se rapprocher de Rossetti. Cela dura jusqu'en 1874, après quoi, et jusqu'à sa mort en 1882, Rossetti, dont la santé mentale était devenue plus que précaire, ne correspondit plus avec Jane que par lettres. On connaît, par contre, fort bien les sentiments de celle-ci vis-à-vis de Morris. Le surlendemain de sa mort, elle déclarait à Wilfrid Scawen Blunt : 'I never loved him'. Comme l'ont remarqué plusieurs biographes, le triangle Morris-Jane-Rossetti n'a pas été sans effet sur la vision de l'amour et des rapports entre les sexes que l'on trouve dans l'œuvre de Morris et l'on est en droit de se demander si le grand libéralisme amoureux dont il fait preuve dans ses écrits n'est pas aussi une façon de légitimer son étrange attitude et son incapacité à trouver une solution pendant toutes les années que dura la relation Jane-Rossetti. On lira dans le présent numéro, pp. 83-96, l'article de Catherine DURIEUX, « Les femmes dans News from Nowhere ".

17. On pourrait en effet considérer que Morris ne faisait que revenir aux inclinations politiques de sa jeunesse. Évoquant sa période à Oxford, il écrira beaucoup plus tard qu'ayant lu Carlyle, Ruskin, Kingsley 'I got into my head some socio-political ideas which would have developed probably but for the attractions of art and poetry'.

18. 'With its literature, Morris had been drinking in the social and religious ethic of the North. In his mind he saw the dream of the free northern communities...as an ideal which contrasted vigorously with the social, political and economic relationships of his own world' (Lloyd Eric GREY, William Morris: Prophet of England's New Order, London: Cassell, 1949, p.125).

19. Cité par J.W. MACKAIL, The Life of William Morris, vol. 1, p. 302.

20. Citons en particulier: The Lesser Arts (1877), The Art of the People (1879), The Beauty of Life (1880), Art and the Beauty of Earth (1881), The Prospects of Architecture in Civilisation (1881), Art under Plutocracy (1883), Art, Wealth and Riches (1883).

21. Les spéculations sur la nature du communisme de Morris remontent au moins à J. Bruce GLASIER, William Morris and the Early Days of the Socialist Movement, London: Longmans, Grenn \& Co, 1921, pp. 142-152 ; formulées de diverses façons, elles n'ont jamais cessé. La position de la critique marxiste semble désormais résumée par l'opinion de A.L. Morton, à l'évidence influencé par l'évolution des vues d'E.P. Thompson entre les deux éditions de son William Morris : Romantic to Revolutionary (1955 et 1977): 'When he encountered the ideas of Marx he had no difficulty in recognising that they were really a more precise and scientific expression of what he had long known in his heart' (A.L. MORTON (ed.), Political Writings of William Morris, London: Lawrence \& Wishart, 1984, p. 17). Récemment Nicholas Salmon est cependant allé plus loin dans la dilution du marxisme de Morris : 'It has been argued that Morris's conversion could not have been achieved without some prior knowledge of scientific socialism... To argue such a case is both to underestimate the sophistication of the 
theoretical conclusions Morris had reached in his pre-socialist writings and to ignore his own unambiguous remarks on the subject'. (Nicholas SALMON, " "The Down-Trodden Radical” : William Morris's PreSocialist Ideology', Journal of the William Morris Society, vol. 13, n³, Winter 1999, p. 41). Il est vrai que si l'on s'en tient à 'How I Became a Socialist', Morris lui-même donne l'impression que ses rapports avec le marxisme tenaient du bricolage idéologique : 'Well, having joined a Socialist body... I put some conscience into trying to learn the economical side of Socialism, and even tackled Marx... I read what I could, and will hope that some information stuck to me from my reading, but more, I must think, from continuous conversation with such friends as Bax and Hyndman and Scheu' (A.L. MORTON, Political Writings of William Morris, p. 242. Reproduit dans News from Nowhere and Other Writings, Clive WILMER (ed.), London: Penguin Books, 1998, p. 380).

22. C'est-à-dire qu'on peut y retrouver l'influence des trois ouvrages de Ruskin déjà cités, ainsi que celle de The Political Economy of Life (1857) et vraisemblablement de Unto This Last (1860-1862) et de Fors Clavigera (1871-1884).

23. Évoquant cette période de sa vie dans 'How I Became a Socialist' (article qui parut dans Justice, le journal de la Social Democratic Federation, le 16 juin 1894), Morris dira: 'I fell into practical Socialism' (A.L. MORTON, Political Writings of William Morris, p. 243. Reproduit dans News from Nowhere and Other Writings, op.cit., p. 380).

24. Dans une lettre du 24 juillet 1884 à Robert Thompson il écrit: 'We cannot turn our people back into Catholic English peasants and guild-craftsmen, or into heathen Norse bonders, much as may be said for such conditions of life' (Philip HENDERSON, The Letters of William Morris to his Family and Friends, p. 208).

25. Ibid., p. 188. La lettre (pp. 183-188) qui est essentiellement 'a rather long-winded sketch of my uneventful life' ( $p .184$ ) est une sorte de première version de 'How I Became a Socialist', essai beaucoup plus connu avec lequel on la confond parfois. Dans celui-ci il ajoute que ce qui, en dernier lieu, acheva de le convertir au socialisme ('put the finishing touch to my conversion') fut sa lecture des critiques de John Stuart Mill à l'encontre du socialisme de Fourier : 'I learned from Mill against his intention that Socialism was necessary' (A.L. MORTON, Political Writings of William Morris, p. 242. Reproduit dans News from Nowhere and Other Writings, op.cit., p. 380). Morris fait référence à la publication posthume dans la Fortnightly Review des Chapters on Socialism de Mill, de février à avril 1879.

26. La mettant parfois au service de son engagement politique : ainsi les Chants for Socialists (des ballades) qu'il commence à rédiger à partir de l'été 1883, et The Pilgrim of Hope (treize monologues en vers publiés dans Commonweal entre avril 1885 et juillet 1886), sorte d'épopée socialiste se déroulant durant la Commune de Paris.

27. Une traduction anglaise ne fut pas publiée avant 1887 et la mort de Marx à Londres en 1883 était passée inaperçue.

28. Paul MEIER, La pensée utopique de William Morris, p. 306.

29. Sauf, on ne le contestera pas, pour ce qui est du processus révolutionnaire enfantant l'Arcadie de Nulle Part. Les chapitres XVII et XVIII de News from Nowhere sont devenus l'ultima ratio de tous ceux qui n'acceptent pas que le marxisme de Morris n'ait pas été d'une absolue pureté. Pour un exemple récent voir Steve Edwards, 'The Trouble with Morris', The Journal of William Morris Studies, vol. $15, \mathrm{n}^{\circ} 1$, hiver 2002, p. 5 : 'Labourism took Morris as one of its standard bearers... News from Nowhere became, for these people, an emblem of a distant future and a signifier of English reasonableness. What had been conveniently forgotten was this text's discussion of revolutionary strategy, its advocacy of insurrectionary violence, and Morris's serious commitment to the self-determination of working-people'.

30. A.L. MORTON, Political Writings of William Morris, pp. 256-257. Ainsi : 'We Socialists cannot forget that these qualities and excellencies [celles de More] meet to produce a steady expression of the longing for a society of equality of condition; a society in which the individual man can scarcely conceive of his 
existence apart from the Commonwealth of which he forms a portion. This, which is the essence of the book, is the essence also of the struggle in which we are engaged'.

31. Dans une lettre adressée le 25 décembre 1884 à J.L. Joynes, ancien professeur d'Eton alors très actif dans le mouvement socialiste, il reproche à Hyndman 'all that insane talk of immediate forcible revolution, when we know that the workers of England are not even touched by the movement', in May MORRIS (ed.), William Morris : Artist, Writer, Socialist, Oxford: Blackwell, 1936, vol.2, p. 590.

32. À l'origine une conférence faite devant la section de Hammersmith de la SDF le 30 novembre 1884. Morris définit ainsi ses 'claims for decent life' : 'First a healthy body; second, an active mind in sympathy with the past, the present, and the future; thirdly, occupation fit for a healthy body and an active mind ; and fourthly, a beautiful world to live in' (A.L. MORTON, Political Writings of William Morris, p. 156).

33. Voir Michael FELLMAN, 'Bloody Sunday and News from Nowhere', Journal of the William Morris Society, vol. 8, n4 Spring 1990, pp. 9-19.

34. Pour cette raison il soutint la grande grève des dockers en août et septembre 1889 (J.W. MACKAIL, The Life of William Morris, vol. 2, pp. 223-225) et en mars 1890 celle des ouvriers de Cours dans le Rhône.

35. Expression qu'il aurait utilisée un soir où la police voulait l'empêcher de parler au coin de Bell Street et d'Edgware Road (selon Stephen COOTE, William Morris : His Life and Work, Stroud: Alan Sutton Publishing, 1996, p. 158).

36. Sur la question de savoir si la révolution sera violente, Morris a évolué. En 1884 il paraît espérer qu'une fois les ouvriers "éduqués » dans les valeurs du socialisme, la société basculera d'elle-même, les exigences du prolétariat entraînant des réformes accélérées aboutissant à un changement qualitatif de société, 'a change in the basis of society' (voir la conclusion de 'How We Live and How We Might Live' dans A.L. MORTON, Political Writings of William Morris, p.158 ; aussi p. 134). Il est déjà conscient que tout processus politico-économique opposant une classe à une autre porte en lui les germes de la violence, mais il veut croire cette dernière incidente à la révolution et non consubstantielle (voir ses lettres du 24 juillet 1884 et du $1^{\mathrm{er}}$ janvier 1885 à Robert Thompson; dans la seconde il écrit 'The last act of it [la révolution] may be civil war'. In Philip HENDERSON, The Letters of William Morris to his Family and Friends, pp. 207 et 228). Après les événements de 1887 il préconisera l'attente et l'éducation idéologique, parlant d'une 'educated revolution', mais il est intéressant de noter que la révolution menant à Nulle Part, bien qu'éclatant en 1952, n'est pas le produit de l'éducation du prolétariat, mentionnée brièvement pour la forme, mais d'une suite pessimiste au scénario de 'How We Live and How We Might Live'. La pression de la classe ouvrière et des syndicats aboutit à un quasi-socialisme d'État qui fonctionne mal, à la résistance du patronat et finalement à la violence et à la guerre civile. Les ouvriers sont surtout poussés par leur «instinct » (le mot est utilisé plusieurs fois), 'a longing for freedom and equality akin if you please to the unreasonable passion of a lover'. L'impression est que c'est dans la guerre et le sang qu'apparaît enfin aux masses la vision d'une société communiste à conquérir, et non au préalable, en raison des décennies passées par quelques-uns à prêcher le socialisme (voir aussi les remarques de John GOODE, 'William Morris and the Dream of Revolution', in John LUCAS (ed.), Literature and Politics in the Nineteenth Century, London: Methuen, 1971, pp. 275-276). Morris n'imagine plus le triomphe du socialisme sans souffrance ni violence. En 1895 encore, il écrit: 'Can that combat be fought out... without loss or suffering? Plainly speaking I know that it cannot'.

37. Le nouveau parti s'installa dans une dépendance de Kelmscott House décorée des portraits de quelques pionniers du socialisme dont, significativement, celui de Thomas More.

38. Contribuant parfois à son périodique Justice auquel il donna en particulier son fragment d'autobiographie politique, 'How I Became a Socialist' en juin 1894.

39. Cité par Ian BRADLEY, William Morris and his World, London: Thames \& Hudson, 1978, p. 91.

40. Et même, pour certains (à commencer par son médecin), d'épuisement au service du socialisme. Selon Mackail, sur Morris qui était de constitution congénitalement fragile, se 
reposait peu et mangeait mal : 'The exposure of the years of the Socialist crusade, when he had perpetually spoken in the open air in all weathers, and in the worse than open air at indoor meetings... told with fatal effect' (J.W. MACKAIL, The Life of William Morris, vol. 2, p. 336).

41. Cité dans Lloyd Eric GREY, William Morris: Prophet of England's New Order, p. 267. La Commune ne pouvait laisser Morris indifférent. Le 18 mars 1886 était publié A Short Account of the Commune of Paris rédigé par Morris, Bax et Victor Dave pour coïncider avec un rassemblement destiné à commémorer la Commune. Morris y rencontra Kropotkine pour la première fois. Dans “ "The Valiant Dead": William Morris and the Paris Commune of 1871', J.B. WRIGHT estime que dans News from Nowhere 'Commune-style revolution, involving the destruction of the capitalist state, is followed by a Commune-style reordering of society' (Journal of the William Morris Society, vol. $13, n^{\circ} 2$, Spring 1999, pp. 34-38).

42. Voir J. Alex MACDONALD, 'The Revision of News from Nowhere', Journal of the William Morris Society, vol. 3, n², Summer 1976, pp. 8-15.

43. On renverra en premier lieu au gros ouvrage de Paul Meier. Voir aussi dans le présent numéro, pp. 25-36, l'article de Michel PRUM, « News from Somewhere: William Morris et l'héritage socialiste britannique ».

44. Morris lut l'ouvrage de Bellamy au début mai 1889. Le 13 il écrivait à Bruce Glasier : 'I wouldn't care to live in such a cockney paradise as he imagines' (Philip HENDERSON, The Letters of William Morris to his Family and Friends, p. 315).

45. Le livre de Bellamy parut en Grande-Bretagne en 1889. Il y eut 17 éditions dans l'année.

46. Voir John GOODE, 'William Morris and the Dream of Revolution', p. 278.

47. Lyman Tower SARGENT, 'William Morris and the Anarchist Tradition', in Florence S. BOOS \& Carole G. SILVER (eds.), Socialism and the Literary Artistry of William Morris, Columbia: University of Missouri Press, 1990, pp. 61-73.

48. Il s'agissait surtout d'anarcho-syndicalistes, 'a curious assortment of cranks, fire-eaters and felons' (E.P. THOMPSON, William Morris : Romantic to Revolutionary, London: Lawrence \& Wishart, 1955, p . 657 ; sur Morris et les anarchistes, voir pp. 638-641).

49. Fibre qu'il perçoit même dans des essais majeurs comme 'The Dawn of a New Epoch', 'The Society of the Future', 'How Shall We Live Then?'.

50. On a déjà noté que Morris fit la connaissance de Kropotkine le 18 mars 1886. Il le rencontra un certain nombre de fois dans les années qui suivirent et l'invita à donner une conférence à Kelmscott House ainsi qu'à écrire pour Commonweal, auquel Kropotkine donna plusieurs articles qui pourraient avoir eu une influence sur News from Nowhere en 1889-1890 (John PAYNE, 'William Morris : " "Back to the Land”, Pessimism and Utopia', Journal of the William Morris Society, vol. 13, $\mathrm{n}^{\circ} 4$, Spring 2000, p. 50 ; mais voir aussi Paul MEIER, La pensée utopique de William Morris, pp. 283-289 et, dans ce numéro, pp. 37-48, Constance BANTMAN, « William Morris anarchiste?»).

51. Sargent aurait pu ajouter l'entraide, valeur essentielle chez Kropotkine et présente dans les plus banals épisodes de la vie quotidienne des utopiens de Morris.

52. John PAYNE, 'William Morris : "Back to the Land", Pessimism and Utopia', p. 51.

53. Karl MANNHEIM, Ideology and Utopia, New York : Harcourt, Brace \& Co, 1954, p. 173.

54. Certains aspects de la société idéale qu'est Nulle Part sont analysés dans le présent numéro par Emmanuel Roudaut (pp. 49-66) et Nathalie Saudo (pp. 67-82).

55. Voir Paul MEIER, La pensée utopique de William Morris, pp. 107-114.

56. Dans une lettre du 13 mai 1885 à Georgiana Burne-Jones, il avait déclaré : 'I have no more faith than a grain of mustard seed in the future history of "civilisation", which I know now is doomed to destruction, and probably before very long: what a joy it is to think of! and how often it consoles me to think of barbarism once more flooding the world... I used really to despair once because I thought what the idiots of our day call progress would go on perfecting itself: happily I know now that all that will have a sudden check' (in J.W. MACKAIL, The Life of William Morris, vol. 2, p. 144-145). 
57. Peter C. GOULD, Early Green Politics : Back to Nature, Back to the Land and Socialism in Britain, Brighton: The Harvester Press, 1988. Voir en particulier les chapitres 2, 3 et 4.

58. Un million d'exemplaires fut vendu en 1894-1895, ce que Morris trouva avec satisfaction révélateur des aspirations profondes du peuple anglais: 'The thousands who have read that book must if they have done so carefully have found out that sometning better is possible to be thought of than the life of a prosperous mill-hand' (cité par E.P. THOMPSON, William Morris : Romantic to Revolutionary, pp. 715-716).

59. On lira dans le présent numéro, pp. 113-122, l'article de Martine FARAUT, 'Robert Blatchford's The Sorcery Shop : Another Socialist Utopia'. Un peu plus avant dans le vingtième siècle, la pensée de Morris et tout particulièrement l'influence de News from Nowhere se retrouvent dans le Guild Socialism de S.G. Hobson et G.D.H. Cole. Sur ce point, et plus généralement sur la contribution de Morris à la pensée socialiste, voir plus loin, pp. 97-111, Valérie AUDA-ANDRÉ, « De l'utopie à la praxis : William Morris et le Guild Socialism ».

60. C'est l'opinion de Bruce GLASIER, William Morris and the Early Days of the Socialist Movement, p. 151.

61. Gilles LAPOUGE, Utopie et civilisation, Paris : Flammarion, 1978, p. 284.

62. Ce qui fait que la dimension mythique du roman n'est pas simplement qu'il est sous-tendu par la longue mémoire de l'âge d'or et du paradis terrestre, mais que le pays de Nulle Part est lui même un mythe au sens où Sorel entendait ce terme: un ensemble d'idées et d'images, d'aspirations morales ou sociales capables de susciter le dévouement, de discipliner le ressentiment, de rassembles les énergies et de déboucher sur un autre monde.

63. Eugene D. LEMIRE, 'Mind in Morris's Englands', Journal of the William Morris Society, vol. 9, $\mathrm{n}^{\circ} 2$, Spring 1991, p. 5 : 'It is not rest that we are moved to by News from Nowhere, but resistance; not a childish absorption in inconsequences that we contemplate, but a constant attempt to stimulate the reader to mature consideration of a different way of looking at humman relations, past and future... The effect of Morris's message is not restfulness, a relaxation of mind, but the kind of heightened awareness that the word "News" in the title tends to excite'. C'est cela, certainement, qui rend compte du fait, à première vue à peine croyable, que G.D.H. Cole, l'un des principaux intellectuels socialistes de la première moitié du vingtième siècle, se convertit au socialisme après une lecture de Nouvelles de Nulle Part: 'I became a Socialist more than fifty years ago when I read News from Nowhere as a schoolboy and realised quite suddenly that William Morris had shown me the vision of a society in which it would be a fine and fortunate experience to live. Needless to say, I have not lived in such a society, or in any even remotely like it; but I count myself not the less fortunate to have been shown that vision; and I can truthfully say that from the day when I first read News from Nowhere my socialist convictions have remained firmly fixed'. William Morris as a Socialist : A Lecture given on $16^{\text {th }}$ January 1957 to the William Morris Society at the Art Workers' Guild by G.D.H. Cole. Transactions of the William Morris Society. London: William Morris Society, 1960, p. 1.

64. Cf. Stephen COLEMAN \& Paddy O'SULLIVAN (eds.), William Morris and News from Nowhere: A Vision for Our Time, Bideford : Green Books, 1990.

65. Voir l'introduction de Clive WILMER à William Morris: News from Nowhere and Other Essays, Harmondsworth : Penguin Books, 1993, pp. xxxviii-xli).

66. A ceux qui aiment les chronologies bien tassées et voudraient suivre Morris à la trace et au jour le jour rappelons l'existence du travail de Nicholas SALMON aidé de Derek BAKER, The William Morris Chronology, Bristol: Thoemmes Press, 1996, 292 pages. 


\section{AUTEUR}

\section{GILBERT BONIFAS}

Université de Nice-Sophia Antipolis 